\title{
RECHERCHES SUR LES LAITS CHAUFFÉS
}

\section{par}

\author{
le Professeur ORLA-JENSEN
}

\author{
Docteur en philosophie et ès sciences, Copenhague.
}

\section{LA PASTEURISATION BASSE.}

On entend par lait de pasteurisation basse tout lait dont les microbes ont été détruits par chauffage sans être sensiblement modifié au point de vue chimique. La pasteurisation basse pourra certainement être exécutée de bien d'autres manières que par les deux méthodes maintenant employées, qui en forment les limites extrêmes, à savoir : une demi-heure de chauffage à $63^{\circ} \mathrm{C}$. - c'est la pasteurisation lente - et un chauffage instantané à $75^{\circ} \mathrm{C}$., appelé stassanisation. J'ai prouvé moi-même que l'on peut atteindre le but par un chauffage de 5 minutes à $68^{\circ} \mathrm{C}$. Quelle que soit la méthode que l'on emploie, le refroidissement doit en tout cas avoir lieu dans un système de tuyaux fermés et stérilisés, car les installations réfrigérantes par "arrosage" du lait sur des surfaces courbes refroidies ne sont pas seulement des sources d'infection, mais elles sont également nuisibles aux vitamines $\mathrm{C}$ du lait, lesquelles; surtout si le lait est chaud, ne supportent pas l'influence de l'air.

En fait d'objection contre les appareils de pasteurisation lente (le nom même l'indique), on peut souligner le temps que prend la pasteurisation du lait. Mais cet obstacle peut être surmonté. Ce qui est plus fâcheux est le fait que j'ai déjà signalé il y a longtemps dans mon ouvrage bactériologie de laiterie (2), qu'il se développe pendant la pasteurisation des bactéries thermophiles dans le lait.

C'est excellent de pouvoir se débarrasser de $99 \%$ de la flore microbienne primitive du lait, mais l'avantage est plus que douteux lorsque l'on reçoit à la place une autre flore de bactéries. De nouvelles recherches faites en Angleterre et en Amérique ont prouvé que si les appareils de pasteurisation lente sont en service continu pendant plusieurs heures, les dernières portions du lait pasteurisé peuvent renfermer de très grandes quantités de bactéries thermophiles, dont l'origine se trouve dans les restes de lait des portions antérieures, qui s'enrichissent de plus en plus en lesdites bactéries thermophiles au fur et à mesure que les appareils sont employés depuis un temps plus long. Le Prof. Breed et P. A. Hanswn (Geneva, Etats-Unis de l'Amérique du Nord) ont trouvé dans de semblables circonstances jusqu'à 50 millions de bactéries thermophiles par $\mathrm{cm}^{3}$ de lait ayant

(1) Paru dans le Volume jubilaire du Professcur Ch. Porcher.

(2) Dairy Bacteriology (la dernière édition de 1931). J. et A. Churehill, édit., Londres. 
subi la pasteurisation basse. C'est naturellement encore pire lorsque les récipients ont une surface rugueuse, bosselée ou avec des soupapes où des restes de lait peuvent s'amasser. Ce n'est pas appétissant, bien que les bactéries thermophiles ne produisent guère de toxines et ne se développent pas à la température du sang, encore moins à la température sous laquelle le lait est conservé. Il faut cependant souligner que ces faits ne sont pas usuels et que généralement le lait de pasteurisation lente renferme très peu de bactéries thermophiles. Néanmoins, ce qui précède parle en faveur des méthodes qui pasteurisent le lait en un temps court, mais à une température plus élevée,

L'avantage de la pasteurisation lente réside cependant dans le fait que si les appareils sont munis de thermographes, on peut toujours être sûr que le lait a été suffisamment chauffé pour détruire les microbes pathogènes. Des écarts de température de courtes durées ne peuvent pas se manifester dans les grandes quantités de lait qui sont traitées ici, et n'ont, du fait de la longue période de chauffage, aucune importance. En réalité, 20 minutes de chauffage à $63^{\circ} \mathrm{C}$. suffisent. Ce n'est que pour plus de sûreté que l'on chauffe pendant 30 minutes, voire 45 minutes, y compris le temps que prend le remplissage et la vidange des appareils. Cette longue période n'est pas profitable au lait, et devrait être diminuée, En somme, peu importe que la température soit pendant une partie du temps à un ou deux degrés au-dessous de $63^{\circ} \mathrm{C}$.

Plus la durée du chauffage est courte, plus les risques de voir passer des microbes pathogènes vivants à travers les appareils sont grands, et si l'on va, comme dans la stassanisation, jusqu'à un chauffage momentané, le danger est particulièrement grand. Les $75^{\circ} \mathrm{C}$. employés représentent de plus une température très critique. Si la température est nettement inférieure, les bactéries pathogènes ne meurent pas, et si la température est nettement supérieure, le lait subit une haute pasteurisation. C'est qu'on exige ici un réglage de la chaleur qu'il n'est pas si facile de maintenir dans la pratique. Pour que les résultats puissent répondre à ceux auxquels on est arrivé dans la laiterie d'essais de l'Etat danois, les tubes de chauffage doivent avoir exactement les mêmes dimensions que ceux employés dans cette laiterie, et le lait doit y être introduit et doit y circuler exactement à la même température et à la même vitesse que dans celle-ci.

Même si ces difficultés peuvent être surmontées, il faut exiger de plus que l'on emploie du lait très propre et non acide pour la stassanisation, car s'il se dépose pendant la pasteurisation des quantités notables des substances de lait, le chauffage deviendra naturellement plus mauvais.

Il y a aussi ici lieu de nommer le fabricant de machines danois, 
M. Jonas Nielsen, qui a fabriqué longtemps avant Stassano des systèmes de tuyaux pour le chauffage et la réfrigération du lait. II est vrai que ces appareils étaient, dans leur forme primitive, destinés à la stérilisation complète du lait, mais dans leur forme actuelle, où le chauffage a lieu au moyen d'eau au lieu de vapeur, ils peuvent également être employés pour la pasteurisation haute et basse.

Déjà en 1905, avant l'apparition de divers appareils américains et allemands de pasteurisation basse, je construisis, en me basant sur mes études des modifications du lait pendant le chauffage, mon appareil de pasteurisation de ménage, simplement composé d'un bain-marie qui contient une quantité suffisante d'eau pour qu'une température d'environ $63^{\circ} \mathrm{C}$. puisse être maintenue pendant une demiheure sans chauffage. Si les bouteilles de lait sont chauffées avec l'eau froide sur une flamme de gaz jusqu'à une température de $65^{\circ} \mathrm{C}$., et si l'on éteint ensuite le gaz, on obtient les températures suivantes :

Immédiatement dans l'eau ........... dans le lait $59^{\circ} \quad 65^{\circ} \quad$ C.

Après 6 minutes dans l'eau $\ldots \ldots \ldots \ldots \ldots .64^{\circ} 1 / 4 \mathrm{C}$. dans le lait $63^{0} 1 / 4 \mathrm{C}$.

Après 15 minutes dans l'eau $\ldots \ldots \ldots \ldots .63^{\circ} \quad 1 / 2 \mathrm{C}$. dans le lait $64^{\circ} \quad \mathrm{C}$.

Après 30 minutes dans l'eau $\ldots \ldots \ldots \ldots \ldots 63^{\circ}$ C. dans le lait $63^{\circ}$ C.

Le lait est donc chauffé de cette façon pendant 25 minutes jusqu'à 63-640 C., température qui détruit tous les mierobes de maladie. Le refroidissement a lieu lorsque l'eau chaude est petit à petit refoulée par l'eau froide. De cette manière, on évite que les bouteilles cassent.

La pasteurisation du lait en bouteilles a été faite sur une grande échelle en Allemagne. On a également fait appel au chauffage du lait avant la mise en bouteilles, avec emploi ultérieur de bouteilles en acier pour activer la vitesse de la pasteurisation et, par suite, le rende ment des appareils. Dans la plupart des cas, ces méthodes ont malheureusement été abandonnées de nouveau, du fait qu'elles sont plus coûteuses que les méthodes par lesquelles le lait est pasteurisé et refroidi avant la mise en bouteilles.

La méthode la plus hygiénique pour pasteuriser le lait est indubitablement de le chauffer en bouteilles, c'est-à-dire en bouteilles de verre sans chauffage préalable. Les avantages sont les suivants :

10 Le lait n'a aucun goût métallique, n'étant pas en contact avec du métal pendant le chauffage.

$2^{\circ}$ Comme le lait n'est pas en contact avec l'air pendant le chauffage, il garde mieux sa teneur naturelle en acide carbonique et en vitamines que par toute autre méthode de pasteurisation basse.

$3^{\circ}$ Comme le lait à l'état chauffé n'est soumis à aucun travail mécanique (remuage, pompage, changement subit de pression, friction, 
etc.), la faculté que sa crème a de monter est moins réduite que par toute autre méthode de pasteurisation basse, ce qui, à son tour, le rend plus apte à subir l'épreuve de ma réaction crémométrique. pour la pasteurisation lente.

$4^{0}$ Toute infection après la pasteurisation du lait est éliminée.

$5^{\circ}$ Le fait que la bouteille est également pasteurisée augmente le pouvoir de conservation du lait.

\title{
DES CRITÉRIUIVS DONT ONTDEVRAIT S'INSPIRER ET DES MÉTHODES QUE L'ON DEVRAIT SUIVRE DANS L'APPRÉCIATION D'UN PROCÉDÉ DE PASTEURISATION DU LAIT
}

\author{
par HENRI STASSANO.
}

En écrivant cet article, je m'adresse à tous ceux qui, par leurs études ou leurs fonctions, sont appelés à se prononcer sur la valeur de tel ou tel procédé, de tel ou tel appareil de pasteurisation du lait, au double point de vue de l'hygiène et de la pratique industrielle, lesquelles, désormais, sont inséparables. Il est, à mon avis, d'une urgente nécessité que les savants et,les spécialistes du lait se mettent d'accord sur le choix des critériums, à la fois scientifiques et pratiques, qui seuls peuvent permettre un jugement sûr, une appréciation valable, impartiale. J'exprime le souhait que le prochain Congrès international de Laiterie mette ce sujet à l'ordre du jour de ses discussions. Déjà, au Congrès de 1931, à Copenhague, soit en séance publique, soit dans des conversations particulières, j'ai appelé l'attention sur la nécessité qu'il y a, d'ores et déjà, d'envisager : $1^{\circ}$ la conservation de l'acide carbonique ; $2^{\circ}$ l'absence concomitante d'aération dans un lait convenablement pasteurisé.

Satisfaire à ces deux observations qui, jusqu'ici, n'ont, pour ainsi dire, jamais été faites, e'est assurer à une pasteurisation du lait réalisée rationnellement deux caractéristiques importantes, mais qui ne sont pas seules, ainsi que nous allons le voir par la suite. Quelques explications feront mieux connaître l'importance de ce que j'avance, sans crainte d'être exposé, lecture faite, au reproche d'exagération ou de partialité.

A. Par la conservation aussi parfaite que possible de l'acide carbonique pendant une pasteurisation bien faite du lait, d'une part, les sels de chaux et les phosphates se maintiennent dans le lait dans leur état antérieur; d'autre part, les équilibres chimiques du lait étant, dans une certaine mesure, sous la dépendance de l'acide carbonique de cette liqueur, si ce gaz n'abandonne point ses labiles combinaisons, le lait conserve toute sa valeur biologique, ainsi que 